\title{
O gerenciamento da produção segundo a Teoria das Restrições: uma aplicação em uma empresa de desenvolvimento de software
}

\begin{abstract}
Ana Maria Magalhães Correia Mestrado em andamento em Engenharia de Produção pela Universidade Federal da

Paraíba - UFPB

Caixa Postal 5045. CEP: 58051-970

E-mail: aninhamagalhaes23@hotmail.com
\end{abstract}

Armistrong Martins da Silva Mestrado em andamento em Engenharia de Produção pela Universidade Federal da

Paraíba - UFPB

Caixa Postal 5045. CEP: 58051-970 E-mail: armistrongmartins@hotmail.com

Ana Carolina Costa de Oliveira Mestrado em andamento em Engenharia de Produção pela Universidade Federal da Paraíba - UFPB Caixa Postal 5045. CEP: 58051-970 E-mail: carolyneoliveira@gmail.com

Antônio de Mello Villar Doutor em Engenharia de Produção pela Universidade Federal da Paraíba - UFPB Caixa Postal 5045 - CEP: 58051-970 E-mail: avillar@ct.ufpb.br

\section{RESUMO}

Este artigo tem por objetivo aplicar os princípios da Teoria das Restrições (TOC) para auxiliar no gerenciamento da produção de uma empresa de desenvolvimento de software, abrangendo questões relacionadas à aplicação dos seus cinco passos e na consequente melhoria dos processos organizacionais. A metodologia utilizada está fundamentada nas pesquisas bibliográfica, descritiva e exploratória, tendo sido levantadas as informações necessárias para atender os objetivos propostos por este estudo. Foram identificadas nas atividades da empresa duas restrições no sistema (conversão de dados e personalizações), e ao final do processo de focalização, com a implantação das melhorias nas atividades, a empresa obteve um aumento de desempenho, refletindo em maiores lucros. Portanto, os resultados indicam que a TOC utilizada como ferramenta de melhoria contínua é bastante útil no gerenciamento da produção, sendo viável e promissora na identificação de ações a serem realizadas para otimização do sistema como um todo.

Palavras-chave: Gerenciamento da Produção. Teoria das Restrições. Desenvolvimento 
O gerenciamento da produção segundo a Teoria das Restrições: uma aplicação em uma empresa de desenvolvimento de software Ana Maria Magalhães Correia, Armistrong Martins da Silva, Ana Carolina Costa de Oliveira, Antônio de Mello Villar

de Software.

The management of production according to the Theory of Constrains: an application in a software development company

\section{ABSTRACT}

This paper aims to apply the principles of Theory of Constrains (TOC) in order to help the management of the production in a software development company, including questions related to implementation of its five stages, results in the improvement of organizational processes. The methodology is based on literature review and descriptive and exploratory research, raised the information necessary to satisfy the objectives proposed by this study. In the activities of the company were identified two constrains on the system (data conversion and customization), and at the end of the focusing process, with the implementation of the improvement of the activities, the company had an increase in performance, reflected in higher profits. Therefore, the results indicate that TOC used as an instrument for continuous improvement is very useful in the management of production and is quite feasible and promising in the identification of actions to optimize the performance of the system as a whole.

Key-words: Production Management. Theory of Constrains. Software Development.

\section{INTRODUÇÃO}

Com o processo de evolução econômica ocorrida a partir da segunda metade do século $\mathrm{XX}$, os sistemas produtivos tiveram que passar por profundas transformações em decorrência das exigências impostas pelo mercado cada vez mais mutável e competitivo. Diante dessas pressões, as organizações têm intensificado seus esforços e investido na incorporação de novas idéias, ferramentas e métodos a fim de aperfeiçoarem seu sistema de gestão e melhorar continuamente seu desempenho produtivo através de decisões que flexibilizem os processos e maximizem os resultados, gerenciando os seus recursos para manter a eficiência e eficácia das operações.

É nesse cenário que a Teoria das Restrições (TOC) surge como uma ferramenta de gestão que auxilia na tomada de decisão perante a identificação dos problemas que 
O gerenciamento da produção segundo a Teoria das Restrições: uma aplicação em uma empresa de desenvolvimento de software Ana Maria Magalhães Correia, Armistrong Martins da Silva, Ana Carolina Costa de Oliveira, Antônio de Mello Villar

estão afetando a melhoria de desempenho nos processos produtivos. Em consonância com a assertiva, Cabral e Fleury (2007) acrescentam que a TOC é uma ferramenta para gerenciamento de sistemas produtivos que trabalha com ações que visam atingir um objetivo específico, denominado meta. Ela disponibiliza um roteiro estruturado, objetivando desenhar cenários, formular estratégias e alicerçar soluções para os gargalos dos sistemas produtivos.

Neste contexto, Tubino (1999) salienta que, como todo sistema produtivo possui algum recurso que limita sua capacidade de produção chamado de gargalo - entendido como um ponto do sistema produtivo (máquina, transporte, espaço, homens, demanda, etc.) que limita o fluxo de itens no sistema - a TOC propõe mudanças, orientando a programação da produção através da restrição, de forma a utilizá-la da maneira mais eficaz possível.

Segundo Cox III e Spencer (2002), a restrição deve ser entendida como o recurso que mais limita a produtividade de uma empresa, funcionando como um entrave para 0 alcance do desempenho satisfatório em relação à meta estabelecida.

Assim, o gerenciamento da produção utilizando a Teoria das Restrições tem seu maior impacto na medida em que permite aos gestores identificar os fatores que estão causando os gargalos e analisar a melhor maneira de intervir e solucioná-los. Desta forma, pode cooperar efetivamente como elemento condutor das análises organizacionais em todos os níveis das atividades da empresa, contribuindo para o processo de tomada de decisão, permitindo o entendimento e a melhoria da organização como um todo em função de seu objetivo final, ou seja, sua meta.

Diante disso, este artigo visa aplicar os princípios da Teoria das Restrições como uma ferramenta auxiliar do gerenciamento da produção numa empresa de desenvolvimento de software, abrangendo questões relacionadas à aplicação das cinco etapas de focalização e na consequente melhoria dos processos organizacionais. Os benefícios esperados dessa aplicação são: i) melhor compreensão de todo o processo produtivo da empresa; ii) uma visão sistêmica dos problemas encontrados; e por conseqüência; iii) um melhor gerenciamento desse processo, buscando priorizar as soluções que melhor se adequem as necessidades da empresa. 
O gerenciamento da produção segundo a Teoria das Restrições: uma aplicação em uma empresa de desenvolvimento de software Ana Maria Magalhães Correia, Armistrong Martins da Silva, Ana Carolina Costa de Oliveira, Antônio de Mello Villar

Logo, este artigo encontra-se dividido em três partes, além desta introdução e das considerações finais. A primeira trata da temática do gerenciamento da produção, a segunda explora a questão da Teoria das Restrições e os aspectos relacionados às melhorias de desempenho, e por fim, a última parte analisa a aplicação da TOC através da focalização do processo em cinco etapas na empresa estudada, descrevendo os métodos e os procedimentos realizados.

\section{EIXO TEMÁTICO}

Na sequência são discutidos alguns temas relevantes à consecução do objetivo proposto, explicitando conceitos de gestão da produção, teoria das restrições (TOC) e medidas de desempenho da teoria das restrições.

\subsection{Gestão da Produção}

A gestão da produção na concepção de Favaretto (2001), diz respeito a um conjunto de processos responsável por todas as atividades da produção, desde a compra da matéria-prima até a expedição. Ou seja, está relacionado à administração de todos os procedimentos e processos direcionados à produção em uma empresa de manufatura.

Segundo Tubino (1997), a função produção é o centro dos sistemas produtivos, responsável por gerar bens ou serviços comercializados pelas empresas, por meio de um ou mais processos de conversão. Diante disso, o gerenciamento da função produção contribui para um eficiente desempenho nas demais funções empresariais e um melhor posicionamento da empresa no mercado. Daí a importância de se ter gestores competentes e preparados para administrar as mudanças e exigências do mercado.

Sob essa ótica, Slack et al. (2001) salientam que, para que a função produção seja eficaz, deve se usar eficientemente seus recursos e produzir bens e serviços de maneira que satisfaça os seus consumidores. Além disso, ela deve ser criativa, inovadora e vigorosa para introduzir novas e melhoradas formas de produção, a fim de 
O gerenciamento da produção segundo a Teoria das Restrições: uma aplicação em uma empresa de desenvolvimento de software Ana Maria Magalhães Correia, Armistrong Martins da Silva, Ana Carolina Costa de Oliveira, Antônio de Mello Villar

proporcionar à organização meios de sobrevivência no longo prazo, gerando vantagem competitiva sobre seus concorrentes.

Assim, um dos principais desafios da gestão da produção na atualidade é concentrar-se na busca por uma alta eficiência dos recursos produtivos como forma de maximizar os rendimentos operacionais, procurando aliar o bom relacionamento com os clientes e fornecedores para a obtenção de vantagem competitiva. Neste contexto, Kopak (2006) acrescenta que, para que os resultados ocorram de maneira positiva é necessário que haja sinergia entre diversas áreas funcionais da empresa, em função da necessidade de troca de informações para a validação das estratégias traçadas pelo planejamento e controle da produção, assegurando que o sistema produtivo possa realizar e atingir as metas da organização. Além do que, o mercado altamente competitivo exige o trabalho cooperativo entre as diversas áreas organizacionais.

De acordo com Nardini e Pires (2002), a gestão da produção numa indústria costuma ser representada, praticamente, pelas atividades de planejamento e controle da produção. Diante disso, o termo Planejamento e Controle da Produção (PCP) é utilizado para designar uma série de atividades, tradicionalmente executadas dentro de uma atividade mais ampla e fundamental dentro da indústria: a gestão da produção. Daí a importância de associar os fundamentos da TOC ao planejamento do PCP.

Corroborando, Villar, et al. (2008) acrescentam que a programação e controle da produção atua como uma função de staff coordenando diversas atividades da área industrial e se constitui na base em que se apóia a maioria dos controles industriais, estando o departamento subordinado diretamente à engenharia. Diferentemente das demais funções de apoio da área industrial, o PCP é uma função administrativa relacionada com o planejamento, direção e controle do suprimento de materiais, peças e componentes e das atividades do processo de produção em uma empresa.

Esse departamento recebe influências tanto do ambiente externo, quanto do ambiente interno da organização. Externamente, destacam-se as políticas econômicas do governo, políticas de produção/vendas da concorrência, fornecedores, leis, dentre outras. No ambiente interno, o PCP recebe influências e informações de outras áreas funcionais, tais como: marketing, vendas, produção, qualidade, contabilidade/finanças, 
O gerenciamento da produção segundo a Teoria das Restrições: uma aplicação em uma empresa de desenvolvimento de software Ana Maria Magalhães Correia, Armistrong Martins da Silva, Ana Carolina Costa de Oliveira, Antônio de Mello Villar

engenharia, recursos humanos e compras; no qual todos os departamentos trabalham sinergicamente entre si, seguindo instruções do PCP, porém, sem necessariamente estarem subordinados diretamente ao mesmo. Nesse sentido, cabe ao PCP buscar um equilíbrio entre os diversos pontos de vista para dar condições de eficiência à empresa como um todo.

Desta forma, a integração das funções de planejamento e programação da produção, concentradas no departamento de PCP e trabalhando em conjunto com outras áreas funcionais da organização, ocasionam melhorias significativas para a eficiência das condições de produção. Segundo Kim et al. (1997), essas melhorias podem ser introduzidas através da redução de conflitos de programação, redução do tempo de fluxo de materiais em processo, aumento da utilização dos recursos de utilização e adaptação a eventos irregulares no chão de fábrica.

Em sua forma mais complexa, Villar et al. (2008) informam que o PCP exerce cinco funções a fim de cumprir sua missão, que são:

a) Gestão de estoques: função através da qual o PCP mantém a produção abastecida de matérias-primas, peças, acessórios, materiais auxiliares, etc.;

b) Emissão de ordens: consiste na preparação do Programa de Produção e na tomada de providências para se ter, a tempo, todos os itens necessários a esse programa, tais como, matérias-primas, peças compradas, peças fabricadas e produtos acabados;

c) Movimentação de ordens: fornece as informações do que foi fabricado e, nos tipos de PCPs mais complexos toma todas as providências para fabricar: retirada de matérias-primas, liberação das ordens de fabricação, contagem das peças, transferências e entrega das peças produzidas, etc.;

d) Programação de ordens de fabricação: consiste numa verdadeira verificação da viabilidade do atendimento das ordens de fabricação, significa à função de preestabelecer a ocasião em que serão executadas as operações de fabricação das peças; 
O gerenciamento da produção segundo a Teoria das Restrições: uma aplicação em uma empresa de desenvolvimento de software Ana Maria Magalhães Correia, Armistrong Martins da Silva, Ana Carolina Costa de Oliveira, Antônio de Mello Villar

e) Acompanhamento da produção: consiste da comparação do que foi programado com o que foi produzido e a ação de corrigir ou fazer corrigir as anomalias entre os dois, ou seja, o gerenciamento dos gargalos.

Diante do exposto, constata-se que um departamento de PCP eficiente assume grande importância no processo de gestão da produção devido às características acima citadas, onde ocorre um ganho de tempo significativo a partir de uma programação confiável com um sequenciamento correto de produção. Uma boa gestão nesses aspectos é vital para que a empresa possa obter vantagem competitiva.

Nestas circunstâncias, muitas empresas se vêem forçadas a rever seu processo de tomada de decisões e repensar como seus sistemas produtivos devem se posicionar estrategicamente para garantir vantagens competitivas sustentáveis frente à concorrência.

Para tanto, os gestores ao acompanhar a produção precisam ter a sensilidade em perceber o momento certo de incorporar novos instrumentos alternativos na tomada de decisão e adotar novas abordagens de gestão na busca por uma maior excelência produtiva, tais como: a Gestão da Qualidade Total (TQM), Gestão Baseada em Atividades (ABC/ABM) e, mais recentemente, a Teoria das Restrições (TOC), que está sendo utilizada como instrumento de gestão para identificação dos fatores que interrompem de alguma forma a eficiência dos fluxos de processo, contribuindo para se alcançar a melhoria contínua e a vantagem competitiva.

A utilização dos fundamentos da Teoria das Restrições é melhor explanada no tópico que segue.

\subsection{Teoria das Restrições - TOC}

A Teoria das Restrições, também denominada TOC (Theory of Constraints), foi inicialmente descrita por um físico israelense chamado Eliyahu M. Goldratt, em seu livro: "A Meta".

A TOC, segundo Dettmer (1997), é uma teoria prescritiva, ou seja, ajuda a identificar o que está impedindo um melhor desempenho da organização e também 
O gerenciamento da produção segundo a Teoria das Restrições: uma aplicação em uma empresa de desenvolvimento de software Ana Maria Magalhães Correia, Armistrong Martins da Silva, Ana Carolina Costa de Oliveira, Antônio de Mello Villar

apresenta o que deve ser feito e a forma de fazê-lo. Wei et al. (2002) concordam que a TOC oferece uma clara orientação para realizar esse reajuste nas atividades.

Dessa forma, essa teoria é considerada um desenvolvimento relativamente recente no aspecto prático da tomada de diversas decisões organizacionais, nas quais existem restrições. Restrição, na concepção de Wanke (2004), pode se entendida como qualquer fator numa empresa que a impede ou limita seu movimento em direção aos seus objetivos. O autor afirma que existem dois tipos básicos de restrições: físicas e não-físicas. As restrições físicas na maior parte das vezes estão relacionadas a recursos: máquinas, equipamentos, veículos, instalações, sistemas etc. As restrições não-físicas podem ser a demanda por um produto, um procedimento corporativo ou mesmo um paradigma mental no encaminhamento de um problema.

Considerando que para a maioria das empresas a meta ou o objetivo principal é o lucro presente e sua sustentabilidade no futuro, a TOC procura administrar adequadamente a produção de forma que os custos sejam mantidos sob controle e os ganhos protegidos. Nesse sentido, a TOC considera que todo sistema deve ter no mínimo uma restrição. Se isso não fosse verdade, então o sistema real deveria ter lucro constante.

Por essa razão, a identificação das restrições representa oportunidades de melhoria, uma vez que ao identificá-las e corrigi-las, permitem alcançar a meta estabelecida pela organização. A TOC, neste caso, apresenta as restrições como algo positivo, já que elas determinam o desempenho do sistema, ou melhor, é a restrição que governa a capacidade do sistema.

Segundo Cox III e Spencer (2002), o gerenciamento de restrições é uma nova abordagem que planeja e controla a produção e venda de produtos, reconhecendo o poderoso papel que a restrição desempenha na determinação da saída do sistema de produção como um todo. Ainda de acordo com os autores, através do conhecimento e da compreensão dos aspectos envolvidos com o gerenciamento das restrições, os gerentes podem perceber melhorias imediatas no resultado de suas organizações e, por meio de uma abordagem focalizada de aprimoramento contínuo, podem planejar para suprir também as necessidades futuras. 
O gerenciamento da produção segundo a Teoria das Restrições: uma aplicação em uma empresa de desenvolvimento de software Ana Maria Magalhães Correia, Armistrong Martins da Silva, Ana Carolina Costa de Oliveira, Antônio de Mello Villar

Gupta et al. (2002) relatam que a TOC busca ajudar os gerentes em todos os níveis da organização para manter um foco apropriado na restrição do sistema. Ou seja, reconhece que a restrição do sistema limita o desempenho do mesmo e propõe-se a fixar princípios e conceitos para gerenciar a restrição. Assim, o principal diferencial da TOC em relação a outras teorias existentes está no fato das ações de gestão estarem concentradas no gerenciamento do recurso de menor capacidade, também chamado de restrição, recurso limitante ou recurso gargalo do sistema.

No processo de tomada de decisões, Wanke (2006) acrescenta que a TOC tem sido aplicada em três diferentes níveis:

- Na gerência da produção; aplicada na resolução de problemas relacionados aos gargalos, à programação e à redução dos estoques;

- Na análise de rentabilidade; levando à mudança de decisões baseadas em custo, para decisões focadas na melhoria contínua das operações que afetam a rentabilidade; e,

- Na gestão de processos; identificando fatores organizacionais, que não são necessariamente recursos, mas que impedem as empresas de atingirem seus objetivos.

Neste contexto, para que a TOC contribua eficazmente para a tomada de decisão, dois aspectos são fundamentais para o seu entendimento:

1) A empresa é um sistema, um conjunto de elementos entre os quais existe uma relação de interdependência, onde "cada elemento depende do outro de alguma forma e assim, o desempenho global do sistema depende dos esforços conjuntos de todos os seus elementos" (CORBETT NETO, 1997);

2) A existência de pelo menos uma restrição. A partir do reconhecimento de que é uma restrição que limita os lucros, os gerentes destas empresas podem perceber e desenvolver mecanismos para gerenciar da melhor maneira possível esta restrição e assim melhorar o desempenho global do sistema. Noreen et al. (1996) afirmam não haver escolhas nesse assunto, isto é, ou o indivíduo controla as restrições, ou elas o controlam, uma vez que as restrições vão determinar a 'saída' (ganho) do sistema, sejam elas reconhecidas e controladas ou não. 
O gerenciamento da produção segundo a Teoria das Restrições: uma aplicação em uma empresa de desenvolvimento de software Ana Maria Magalhães Correia, Armistrong Martins da Silva, Ana Carolina Costa de Oliveira, Antônio de Mello Villar

Desse modo, para alcançar os objetivos organizacionais todos os membros e setores devem trabalhar de forma sistêmica em detrimento dos benefícios globais. Entretanto, considerando que numa linha de produção sempre existirão recursos com capacidades produtivas diferenciadas, sendo denominado de recurso gargalo aquele cuja capacidade de produção é menor ou igual à demanda por ele existente, para atingir à meta é necessário identificar e corrigir os fatores que estão dificultando a evolução dos processos de forma eficiente.

Sendo assim, partindo da premissa de que a empresa opera com algum tipo de restrição, Goldratt (1992) formula um processo geral de tomada de decisão empresarial, denominado Processo de Aprimoramento Contínuo, composto por cinco passos de focalização apresentados da seguinte maneira:

a) Identificar a(s) restrição(ões) do sistema: onde o recurso de menor capacidade define a capacidade máxima de todo o sistema. Nessa etapa é importante identificar as restrições existentes no sistema, pois não há como aumentar o desempenho do mesmo sem a identificação do seu elo mais fraco, que é o fator deficiente;

b) Decidir como explorar a (s) restrição (ões) do sistema: Nesta etapa a idéia é maximizar o desempenho do sistema e explorar ao máximo a capacidade dos recursos, considerando a restrição, ou seja, obter o melhor resultado possível dentro dessa condição. Para isso, deve-se calcular a rentabilidade por unidade de recurso consumida na restrição. Segundo Wanke (2006), este valor é obtido pela divisão da rentabilidade ou margem de contribuição unitária pelo consumo de recursos da restrição para produzir um produto. Neste caso, a chave para maximizar o lucro é concentrar na produção e na comercialização de produtos com a maior rentabilidade por unidade de recurso consumida na restrição;

c) Subordinar o sistema a restrição: De acordo com Wanke (2006), os recursos e estoques devem ser gerenciados de modo a prover exatamente o necessário para atingir os objetivos definidos para a restrição. Este passo pode implicar na ociosidade de recursos que não são restrições. Desta forma, o objetivo é subordinar todas as decisões à decisão anterior. Ou seja, como o desempenho do sistema como um todo é determinado pelos recursos restritivos (gargalos), isso significa que 
O gerenciamento da produção segundo a Teoria das Restrições: uma aplicação em uma empresa de desenvolvimento de software Ana Maria Magalhães Correia, Armistrong Martins da Silva, Ana Carolina Costa de Oliveira, Antônio de Mello Villar

os demais recursos devem trabalhar no ritmo da restrição, nem mais rápido nem mais lento. Não se pode deixar faltar material para a restrição trabalhar, pois dessa forma ela pode parar, afetando negativamente o sistema como um todo. Por outro lado, também não se deve produzir muito mais do que a restrição consegue processar, pois segundo Elmaghraby et al. (2003), a subordinação à restrição, quando posível, normalmente vem com um custo, uma vez que envolve um aumento nos recursos utilizados;

d) Elevar a (s) restrição (ões) do sistema: Segundo Cox III e Spencer (2002), se após a terceira etapa permanecer alguma restrição, deve-se elevar ou superar essa restrição, acrescentando maior quantidade do recurso escasso no sistema. Significa investir mais na restrição, seja adquirindo mais uma máquina, contratando pessoas, aumentando os turnos de trabalho, entre outros. Dessa forma, a restrição será rompida e o desempenho da empresa subirá até certo índice, quando será limitada por algum outro fator. Assim, a restrição foi mudada e existe agora um novo recurso restritivo a limitar o desempenho da empresa;

e) Se nos passos anteriores uma restrição for quebrada, volte ao passo 1 mas não deixe que a inércia se torne uma restrição do sistema: Mesmo concluídas as etapas anteriores, sempre haverá espaço para aprimoramentos. Se no passo anterior uma restrição foi superada, deve-se voltar à primeira etapa, e iniciar tudo novamente, pois com certeza haverá uma nova restrição a ser quebrada. Nessa etapa deve-se ter cuidado para que a inércia não se torne uma restrição do sistema. Portanto, a empresa precisa saber como trabalhar com as restrições, devendo-se observar que a implementação da TOC pode exigir uma mudança substancial na maneira como a empresa opera. Ignorá-las seria um grande erro. Por isso, é preciso reconhecê-las e administrá-las a fim de que se tornem uma grande oportunidade para alavancar o seu negócio.

Goldratt (1992, p.50) argumenta: 
O gerenciamento da produção segundo a Teoria das Restrições: uma aplicação em uma empresa de desenvolvimento de software Ana Maria Magalhães Correia, Armistrong Martins da Silva, Ana Carolina Costa de Oliveira,

Antônio de Mello Villar

Tente medir por três ou mais medidas não financeiras e você basicamente terá perdido todo o controle. As medidas não financeiras são equivalentes à anarquia. Você simplesmente não pode comparar maçãs, laranjas e bananas e definitivamente não relacioná-las com o resultado final! A meta é fazer dinheiro. Cada medida deve, por definição, conter o significado de dinheiro.

Neste contexto, de acordo com Silva et al. (2005), as medidas de desempenho são sinais vitais da organização que qualificam e quantificam o modo como as atividades de um processo atingem suas metas. Assim, os autores classificam um sistema de medição de desempenho como um conjunto de medidas referentes à organização como um todo (divisões, departamento, etc.) e aos seus processos, de forma a refletir certas características do desempenho para cada nível gerencial interessado. Destarte, pode-se inferir que o desempenho de um produto ou de um serviço dependerá de como cada um satisfaz os requisitos, as necessidades e as expectativas dos clientes.

Para Kopak (2006), o sistema de medidas de desempenho é também chamado de Contabilidade de Ganhos. As medidas utilizadas por esse sistema para avaliar o desempenho são: Ganho, Investimento e Despesa Operacional. Essas medidas são traduzidas nas medidas globais da empresa, como: lucro líquido, retorno sobre o investimento e fluxo de caixa.

Segundo Bornia (2009, p. 165),

uma diferença importante entre o TOC (ganhos) e a abordagem tradicional (custos) é a prioridade dada a cada medida em esforços para melhorar a situação econômica da empresa. $\mathrm{Na}$ abordagem tradicional, o primeiro pensamento do gerente é reduzir os custos (despesas operacionais) normalmente iniciando pela eliminação da mão-de-obra. Após isso, tenta-se diminuir os estoques (inventários) e, só depois, vem à procura por novas oportunidades de aumentar as vendas. Para a TOC, a prioridade é aumentar o ganho com a busca de novos mercados e alternativas, mantendo-se a estrutura (inclusive mão-de-obra) atual. Em segundo lugar, pensa-se na redução dos inventários e, por último, na diminuição da despesa operacional. 
O gerenciamento da produção segundo a Teoria das Restrições: uma aplicação em uma empresa de desenvolvimento de software Ana Maria Magalhães Correia, Armistrong Martins da Silva, Ana Carolina Costa de Oliveira, Antônio de Mello Villar

Entretanto, é importante salientar, segundo os princípios defendidos por Goldratt (1992), que tentar medir o desempenho de uma empresa por três ou mais medidas não financeiras pode acarretar em descontrole. Como a meta da empresa segundo ele, é fazer dinheiro, então cada medida deve ser expressa em resultado monetário.

Nessa disposição, Rogers e Reis (2005) complementam que a TOC define que apenas o Lucro Líquido (LL), o Retorno Sobre o Investimento (RSI) e o fluxo de caixa são parâmetros norteadores do grau de alcance da meta. O Lucro Líquido é uma medida absoluta representando quanto dinheiro a empresa está gerando, diferentemente como definido pela contabilidade. O Retorno Sobre Investimento mensura relativamente 0 Lucro Líquido pela quantidade de investimento absorvido pela empresa. O fluxo de caixa é um parâmetro de sobrevivência.

Contudo, Goldratt e Cox (1997) salientam que as medidas acima descritas estão voltadas para a mensuração do desempenho global da empresa necessitando, no entanto, o estabelecimento de parâmetros que guiem as ações operacionais no sentido do cumprimento da meta. Assim, ou autores definem os seguintes parâmetros operacionais:

- Ganho (G): é definido como índice pelo qual o sistema gera dinheiro através das vendas;

- Inventário (I): é todo dinheiro que o sistema investe na compra de objetos que ele pretende vender;

- Despesa Operacional (DO): é definida como todo dinheiro que o sistema gasta para transformar o inventário em ganho.

Para cálculo do Lucro Líquido (LL), conforme Goldratt (1992), a fórmula é "LL é igual a Margem de Contribuição Total (MCT) ou Ganho Total menos a DO". Onde a DO, refere-se toda a parte fixa da empresa, seja na parte industrial, seja na parte administrativa. Para calcular o Retorno Sobre o Investimento (RSI), divide-se o resultado obtido com o Ganho menos as Despesas Operacionais sobre o Inventário.

Outro fator importante na avaliação do desempenho é a Margem de Contribuição, uma vez que a sua análise propicia informações ao gerente para decidir se é coerente diminuir ou expandir uma linha de produção, para avaliar as alternativas 
O gerenciamento da produção segundo a Teoria das Restrições: uma aplicação em uma empresa de desenvolvimento de software Ana Maria Magalhães Correia, Armistrong Martins da Silva, Ana Carolina Costa de Oliveira,

Antônio de Mello Villar

provenientes da produção, de propagandas especiais, entre outros. Também é possível decidir sobre estratégias de preço, serviços ou produtos e, principalmente, avaliar o desempenho da empresa.

Diante do exposto, o desempenho consistente de algumas organizações está relacionado à forma como as estratégias e as metas são definidas pela organização e como elas orientam os esforços para garantir o sucesso organizacional. Tais estratégias permitem gerar metas através de reuniões com a gerência, que reflitam ou traduzam, de forma equilibrada, os anseios da organização (sua visão e sua missão). A conscientização da alta administração sobre a importância da medição do desempenho e do potencial para a melhoria da gestão é fundamental, visto que, um sistema de medição de desempenho reflete o modo de gerenciamento da organização, sendo um dos pilares da TOC nos procedimentos para o gerenciamento da produção no contexto das restrições e que auxilia os gestores na otimização do desempenho da produção para alcançar as metas organizacionais.

\section{METODOLOGIA}

A metodologia utilizada neste artigo foi fundamentada a partir da pesquisa bibliográfica, cuja natureza é descritiva e exploratória. Foram levantadas informações sobre o assunto em pauta cuja realização se deu numa empresa de desenvolvimento de software. Desta forma, os dados foram levantados no período de março de 2007 a maio de 2009 e as informações obtidas serviram de objeto para análise deste artigo.

Os dados foram extraídos diretamente na empresa, mediante a aplicação de um formulário de entrevistas com os gerentes responsáveis pelo setor financeiro, além de três diretores. Os dados secundários também foram registrados, avaliando-se documentos pertinentes contidos na empresa. O quadro 1 apresenta os instrumentos e métodos de coleta de dados aplicados na pesquisa. 
O gerenciamento da produção segundo a Teoria das Restrições: uma aplicação em uma empresa de desenvolvimento de software Ana Maria Magalhães Correia, Armistrong Martins da Silva, Ana Carolina Costa de Oliveira, Antônio de Mello Villar

\begin{tabular}{|c|c|c|}
\hline $\begin{array}{c}\text { Instrumento de } \\
\text { coleta de dados }\end{array}$ & Tipos & Material de apoio \\
\hline Entrevista & (1) Semi-estruturada. & $\begin{array}{c}\text { (1) Correspondência eletrônica; (2) } \\
\text { Mensagens eletrônicas instantâneas; (3) } \\
\text { Telefone; (4) Anotações. }\end{array}$ \\
\hline $\begin{array}{c}\text { Análise de } \\
\text { documentos }\end{array}$ & $\begin{array}{c}\text { (1) Atas de reuniões; (2) Banco de } \\
\text { dados; (3) Registro de } \\
\text { procedimentos da área operacional. }\end{array}$ & $\begin{array}{c}\text { (1) Fotocópias; (2) Anotações; (3) } \\
\text { Planilhas eletrônicas; (4) Arquivos } \\
\text { informatizados em geral. }\end{array}$ \\
\hline
\end{tabular}

Quadro 1 - Visão geral dos métodos de coleta de dados

Fonte: Elaboração própria (2009).

A pesquisa documental foi realizada nas fichas de produção das atividades onde foram obtidos o sequenciamento de produção e o tempo de cada processo de produção; foram analisados também os relatórios financeiros contendo informações sobre despesas operacionais, dados de custos diretos, custos indiretos, custos variáveis e preço de venda do produto. Em seguida, esses dados foram trabalhados e apresentados em planilhas eletrônicas para facilitar a visualização e um melhor entendimento dos resultados alcançados.

\section{CARACTERIZAÇÃO DA EMPRESA}

A empresa de desenvolvimento de software foi criada em 1995 no estado do Mato Grosso. Iniciou suas atividades com o objetivo de trazer ao estado o que havia de mais moderno em sistemas de gestão empresarial. A empresa trabalha com produtos personalizados com o intuito de maximizar a adaptação do software às necessidades de cada cliente. Para isso conta com o apoio de dois setores: O suporte, representado por uma equipe de profissionais especializados que proporcionam um atendimento às necessidades dos clientes e o desenvolvimento, cuja equipe é qualificada para criações de soluções e inovações tecnológicas nos softwares personalizados. A identificação da empresa nesse artigo foi omitida a pedido da mesma. 
O gerenciamento da produção segundo a Teoria das Restrições: uma aplicação em uma empresa de desenvolvimento de software Ana Maria Magalhães Correia, Armistrong Martins da Silva, Ana Carolina Costa de Oliveira, Antônio de Mello Villar

\subsection{Características do processo produtivo da empresa}

A empresa possui três diferentes produtos. Os softwares comercializados estão descritos a pedido da empresa como produto $X, Y, Z$ :

- $\mathbf{X}$ : É um sistema genérico para gestão empresarial adequado para pequenas e médias empresas, sendo constituído pelos seguintes módulos: cadastro de clientes, cadastro de produtos, cadastro de fornecedor, cadastro de funcionários, controle de estoque, contas a pagar, contas a receber, frente de loja e relatórios padronizados;

- Y: É um sistema de automação comercial voltado para o comércio varejista e atacadista desenvolvido para absorver as necessidades do mercado de venda, auxiliando o controle administrativo, financeiro e alguns relatórios customizados;

- Z: É um sistema para gestão empresarial adequado para grandes empresas, possui dicionário de dados o que possibilita a total customização das necessidades da empresa. Através da análise dos sistemas comercializados optou-se por estudar o processo produtivo do produto $Z$, pois os produtos $X$ e $Y$ são pacotes fechados enquanto que o $Z$ necessita do desenvolvimento de um projeto de customização. Seu processo produtivo está representado no macro-fluxograma (Figura 2).

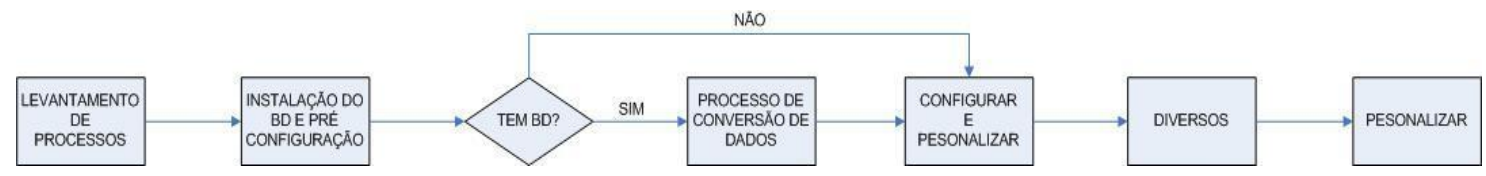

Figura 2 - Macro-fluxograma do produto Z

Fonte: Elaboração própria (2009).

A primeira fase dessa etapa produtiva é o levantamento de processos, no qual são identificados o funcionamento da empresa do cliente, detalhamento das rotinas internas, reunião com o setor estratégico da empresa para criação e modificação das rotinas. Em seguida é realizada a instalação de banco de dados e préconfigurações.

A próxima fase do processo consiste em verificar se a empresa tem banco de dados. Caso ela possua algum software será necessária à conversão do banco de 
O gerenciamento da produção segundo a Teoria das Restrições: uma aplicação em uma empresa de desenvolvimento de software Ana Maria Magalhães Correia, Armistrong Martins da Silva, Ana Carolina Costa de Oliveira, Antônio de Mello Villar

dados para a nova ferramenta. Caso contrário, o procedimento seguirá para a próxima fase.

Em configurar e personalizar, o software será configurado com os principais dados da empresa, os procedimentos levantados na primeira fase serão implementados e o software já personalizado será instalado na empresa. Posteriormente na fase de diversos, é realizado o treinamento e possíveis alterações, no qual os funcionários serão instruídos para utilização do software personalizado.

Finalmente, na fase de personalização, será realizada uma reunião de implantação, apresentando os resultados do software personalizado, detalhando todas as rotinas implementadas e justificativas das possíveis restrições do sistema. Todas as atividades do desenvolvimento de software são realizadas seqüencialmente, de forma que, em média, um projeto é desenvolvido em 5,23 meses.

Desta forma, o desenvolvimento do software personalizado deve ser realizado por uma equipe de trabalho composta por: um consultor, dois desenvolvedores, dois analistas e três técnicos, os quais possuem um regime de trabalho de 32 horas semanais.

\subsection{Aplicação prática}

A abordagem proposta para este trabalho fundamenta-se no processo de melhoramento do gerenciamento da produção a qual objetiva: reduzir os gastos e desperdícios, aumentar a qualidade, aprimorar os produtos oferecidos na empresa, aumentar a produtividade e satisfazer os clientes. Estas ações na empresa estão direcionadas com a gestão da produção atribuída ao PCP e, posteriormente, com os cinco passos de focalização da TOC.

Nesse sentido, conforme visto no eixo temático, a integração das funções de planejamento e programação da produção, concentradas no departamento de PCP e trabalhadas em conjunto com outras áreas funcionais da organização, ocasionam melhorias significativas para a eficiência das condições de produção da empresa e estão descritas a seguir: 
O gerenciamento da produção segundo a Teoria das Restrições: uma aplicação em uma empresa de desenvolvimento de software Ana Maria Magalhães Correia, Armistrong Martins da Silva, Ana Carolina Costa de Oliveira, Antônio de Mello Villar

a) Gestão de estoques: Nesse caso, como a empresa trabalha com o desenvolvimento de softwares, ou seja, serviço não existe estoque e consequêntemente o gerenciamento do mesmo;

b) Emissão de ordens: Como o produto $Z$ é um produto produzido por encomenda, a emissão das ordens de produção acontece a partir da autorização do cliente para a execução do serviço;

c) Movimentação de ordens: A movimentação das ordens de produção acontece no processo produtivo já descrito no macro-fluxograma do produto $Z$, através de suas atividades de levantamento de processos, instalação de banco de dados e préconfigurações, verificação se a empresa do cliente possui banco de dados, configuração e personlização, diversos e por fim, a personalização final onde são apresentados os resultados do software personalizado, detalhando todas as rotinas implementadas e as justificativas das possíveis restrições do produto;

d) Programação de ordens de fabricação: A programação da ordem de produção é realizada a partir dos tempos definidos em cada etapa do desenvolvimento do produto Z. Todas as atividades do desenvolvimento de software são realizadas seqüencialmente, de forma que, em média um projeto é desenvolvido em 5,23 meses. Desta forma, o desenvolvimento do software personalizado deve ser realizado por uma equipe de trabalho composta por: um consultor, dois desenvolvedores, dois analistas e três técnicos, os quais possuem um regime de trabalho de 32 horas semanais;

e) Acompanhamento da produção: $O$ acompanhamento da produção é realizado através da execução das etapas de focalização da TOC, descritas a seguir:

\section{Passo 1 - Identificar a(s) restrição(ões) do sistema:}

Essa primeira etapa consiste em identificar a restrição no sistema que limita o ganho. Assim, como sempre haverá uma restrição que impedirá o aumento dos ganhos, a forma mais adequada encontrada para identificação deste recurso na empresa é através do delineamento das atividades para a execução do produto $Z$, conforme tabela 1. A demanda para este produto é de 3 unidades por ano e este, é comercializado ao preço de $R \$ 1.000 .000,00$. 
O gerenciamento da produção segundo a Teoria das Restrições: uma aplicação em uma empresa de desenvolvimento de software Ana Maria Magalhães Correia, Armistrong Martins da Silva, Ana Carolina Costa de Oliveira, Antônio de Mello Villar

Tabela 1 - Quantidade de horas x recursos

\begin{tabular}{|c|c|c|c|c|c|c|}
\hline Nome da atividade & Produto & Demanda & $\begin{array}{c}\text { Tempo } \\
\text { (minutos) }\end{array}$ & $\begin{array}{c}\text { TempoTotal } \\
\text { (minutos) }\end{array}$ & $\begin{array}{c}\text { Disponível } \\
\text { (minutos) }\end{array}$ & $\begin{array}{c}\% \\
\text { Ocupação }\end{array}$ \\
\hline $\begin{array}{l}\text { Levantamento de } \\
\text { processos }\end{array}$ & \multirow{6}{*}{ Z } & \multirow{6}{*}{3} & 4.220 & 12.660 & 14.850 & 85,25 \\
\hline $\begin{array}{l}\text { Instalação banco de } \\
\text { dados e pré- } \\
\text { configurações }\end{array}$ & & & 342 & 1.026 & 2.160 & 47,50 \\
\hline Conversão de dados & & & 4.080 & 12.240 & 10.545 & 116,07 \\
\hline $\begin{array}{l}\text { Configurações e } \\
\text { personalizações }\end{array}$ & & & 7.796 & 23.388 & 25.845 & 90,49 \\
\hline Diversos & & & 1.985 & 5.955 & 7.245 & 82,19 \\
\hline Personalizações & & & 21.030 & 63.090 & 61.275 & 102,96 \\
\hline
\end{tabular}

Fonte: Dados da Pesquisa (2009).

As atividades de conversão de dados com a utilização de 12.240 minutos gastos com disponibilidade de apenas 10.545 minutos e a atividade de personalizações com a utilização de 63.090 minutos gastos com disponibilidade de apenas 61.275 minutos, são as restrições do sistema com 116,07 \% e 102,96\% respectivamente de ocupação, assim compondo-se o mix de produção das atividades com a capacidade disponível.

Observa-se que as demais atividades do processo não apresentam restrição alguma, pois as atividades são executadas com o tempo previsto, portanto, possui produção de 3 unidades por ano (100\% da demanda). Entretanto, como o produto $Z$ para sua finalização necessita da completa execução de todas as suas atividades e visto que as atividades de conversão de dados e personalizações têm sua produção limitada pela restrição, logo a produção do produto $Z$ será parcialmente atendida em 2 unidades por ano $(66,67 \%$ da demanda).

Como a capacidade da restrição determina a capacidade do sistema, calcula-se o lucro máximo anual desta empresa através da metodologia da TOC. Inicia-se calculando a margem de contribuição (throughput) do produto Z. Tendo a margem de contribuição, está é multiplicada pela demanda do mercado, e o resultado é o chamado ganho. Em seguida subtraem-se as despesas operacionais que totalizam $R \$$ 510.915,29, dando-se ao resultado a denominação de lucro máximo. 
O gerenciamento da produção segundo a Teoria das Restrições: uma aplicação em uma empresa de desenvolvimento de software Ana Maria Magalhães Correia, Armistrong Martins da Silva, Ana Carolina Costa de Oliveira, Antônio de Mello Villar

Martins (2001) afirma que a margem de contribuição é a diferença entre a receita e o custo variável de cada produto, ou seja, é o valor que cada unidade efetivamente traz à empresa de sobra entre sua receita e o custo que de fato provocou. Para a Teoria das Restrições o custo variável é o material direto aplicado ao produto. $\mathrm{Na}$ empresa estudada, o valor do material direto é $R \$ 42$. 646,96, como mostra a tabela 2 .

Tabela 2 - Margem de contribuição unitária (throughout)

\begin{tabular}{|c|c|c|c|c|c|}
\hline Produto & Demanda & $\begin{array}{c}\text { Preço de } \\
\text { Venda } \\
\text { (R\$) }\end{array}$ & $\begin{array}{c}\text { Material } \\
\text { direto } \\
(\mathbf{R} \$)\end{array}$ & $\begin{array}{c}\text { Margem de Contribuição } \\
\text { (R\$) }\end{array}$ & $\begin{array}{c}\text { Lucro } \\
\text { (R\$) }\end{array}$ \\
\hline \multirow[t]{4}{*}{ Z } & 2 & $1.000 .000,00$ & $42.646,96$ & $957.353,04$ & $1.914,796,08$ \\
\hline & & & & Ganho & $1.914,796,08$ \\
\hline & & & & Despesas Operacionais & $510.915,29$ \\
\hline & & & & Lucro Máximo Anual & $1.657 .944,51$ \\
\hline
\end{tabular}

Fonte: Dados da Pesquisa (2009).

Portanto, o Lucro Máximo Anual obtido é de $R \$ 1.657 .944,51$. São aplicados a seguir os outros passos da TOC.

\section{Passo 2 - Decidir como explorar as restrições do sistema:}

A segunda etapa consiste em aproveitar a capacidade existente na restrição, que frequentemente é desperdiçada por causa da utilização de regras e procedimentos inadequados para programar e controlar a restrição (COX III; SPENCER, 2002).

Caso não houvesse restrição interna, o mercado em último caso seria a restrição do sistema. Porém, como identificado na etapa anterior, a empresa apresenta duas restrições e nesse caso, segundo Noreen, Smith e Mackey (1996), as decisões devem ser tomadas priorizando atividades prováveis, segundo a quantidade de ganho que produzem por unidade do recurso com restrição de capacidade.

Assim, parte-se agora para o segundo passo do processo da TOC, ou seja, utilizar as restrições encontradas da forma mais eficiente possível. Deve-se descobrir qual dos produtos é o que possui a maior margem líquida proporcionalmente ao fator restritivo, ou seja, determinar qual das atividades contribuem mais para o lucro da empresa, como demonstrado na tabela 3. 
O gerenciamento da produção segundo a Teoria das Restrições: uma aplicação em uma empresa de desenvolvimento de software Ana Maria Magalhães Correia, Armistrong Martins da Silva, Ana Carolina Costa de Oliveira, Antônio de Mello Villar

Tabela 3 - Margem líquida unitária em relação à utilização do fator restritivo

\begin{tabular}{|c|c|c|c|c|}
\hline Atividade & $\begin{array}{c}\text { Tempo } \\
\text { (minutos) }\end{array}$ & $\begin{array}{c}\text { Quantidade } \\
\text { produzida em 60 } \\
\text { minutos }\end{array}$ & $\begin{array}{c}\text { Margem de } \\
\text { contribuição } \\
\text { (R\$) }\end{array}$ & Ganho (R\$) \\
\hline $\begin{array}{c}\text { Conversão de } \\
\text { dados }\end{array}$ & 12.240 & 0,00490 & $957.353,04$ & $4.691,02$ \\
\hline Personalizações & 63.090 & 0,00095 & $957.353,04$ & 909,49 \\
\hline
\end{tabular}

Fonte: Dados da Pesquisa (2009).

Nota-se que entre as atividades que apresentam restrições, a atividade de conversão de dados é a que apresenta um maior ganho ( $R \$ 4.691,02)$ em relação a outra atividade.De posse destas informações, em reunião com a diretoria, algumas ações foram adotadas principalmente na atividade de conversão de dados, com o objetivo de explorar a capacidade da restrição para elevar o ganho da empresa. São elas: i) manutenção preventiva dos equipamentos utilizados no processo produtivo; ii) adoção de procedimentos de controle de qualidade para eliminar defeitos antes e depois da execução das atividades; iii) ativação das restrições em intervalos como lanches, almoço, e mudanças de turno, com funcionários extras para a substituição; e iiii) realização de mudanças de funcionários nas etapas do processamento das atividades que apresentaram as restrições, obtendo com isso, ganhos de tempo essenciais para a otimização do processo, como demonstrado na tabela 4. 
O gerenciamento da produção segundo a Teoria das Restrições: uma aplicação em uma empresa de desenvolvimento de software Ana Maria Magalhães Correia, Armistrong Martins da Silva, Ana Carolina Costa de Oliveira,

Tabela 4 - Mudança estratégica de recursos

\begin{tabular}{|c|c|c|c|c|}
\hline Nome da atividade & $\begin{array}{c}\text { Recursos } \\
\text { (funcionários) }\end{array}$ & $\begin{array}{l}\text { Mudança } \\
\text { estratégica }\end{array}$ & $\begin{array}{c}\text { Tempo } \\
\text { gasto } \\
\text { (minutos) }\end{array}$ & $\begin{array}{l}\text { Tempo gasto } \\
\text { com a mudança } \\
\text { (minutos) }\end{array}$ \\
\hline \multicolumn{5}{|c|}{ Conversão de Dados } \\
\hline $\begin{array}{c}\text { - Avaliar estrategicamente os } \\
\text { dados }\end{array}$ & T1 & \multirow[t]{3}{*}{$\mathrm{A} 1, \mathrm{~A} 2$} & \multirow{8}{*}{12.240} & \multirow{8}{*}{7.650} \\
\hline $\begin{array}{l}\text { - Identificar dados para } \\
\text { converter }\end{array}$ & T2 & & & \\
\hline $\begin{array}{l}\text { - Examinar a estrutura dos } \\
\text { dados }\end{array}$ & T3 & & & \\
\hline - Adequação no BD atual & C1 & $\mathrm{C} 1, \mathrm{C} 2$ & & \\
\hline $\begin{array}{l}\text { - Criar layout/Scripts de } \\
\text { conversão }\end{array}$ & $\mathrm{A} 1, \mathrm{~T} 1$ & $\mathrm{D} 1, \mathrm{C} 1$ & & \\
\hline - Converter BD & T2 & $\mathrm{T} 1, \mathrm{~T} 2$ & & \\
\hline - Validar conversão - Técnico & T2 & $\mathrm{T} 1, \mathrm{~T} 2$ & & \\
\hline - Validação conversão - Cliente & $\mathrm{C} 1, \mathrm{~A} 1$ & $\mathrm{C} 1, \mathrm{~A} 1$ & & \\
\hline \multicolumn{5}{|l|}{ Personalizações } \\
\hline - Personalizações do sistema & A1 & $\mathrm{A} 1, \mathrm{~A} 2$ & \multirow{7}{*}{63.090} & \multirow{7}{*}{55.530} \\
\hline - Personalizações de regras & D1 & D1,D2 & & \\
\hline - Consultas personalizadas & C1 & C1 & & \\
\hline - Relatórios personalizados & $\mathrm{A} 1, \mathrm{~T} 1$ & $\mathrm{~A} 1, \mathrm{~T} 1, \mathrm{~T} 2$ & & \\
\hline - Treinamentos - implantação & $\mathrm{A} 1$ & $\mathrm{~A} 1$ & & \\
\hline - Acompanhamento & $\mathrm{A} 2$ & $\mathrm{~A} 2$ & & \\
\hline - Reunião Final & $\mathrm{C} 1, \mathrm{~A} 1$ & $\mathrm{C} 1, \mathrm{~A} 1$ & & \\
\hline
\end{tabular}

Fonte: Dados da Pesquisa (2009).

Como pode ser observado na tabela acima, nas três primeiras etapas da atividade de conversão de dados foi realizada uma mudança de 3 técnicos (T) para 2 analistas (A), visto que possuem experiência e dinamismo para realizar essas atividades em menos tempo. Na quarta etapa foi acrescido 1 consultor (C); na quinta em vez de 1 analista e 1 técnico, 1 desenvolvedor (D) e 1 consultor (C). Na sexta e sétima etapa, foi acrescido mais 1 técnico e a última, permaneceu com os mesmos funcionários. Já na atividade de personalizações, a mudança na primeira e segunda etapa se deu com um acréscimo de 1 analista e 1 desenvolvedor respectivamente; a terceira etapa permaneceu com 1 consultor. Na quarta etapa houve um acréscimo de um técnico e as demais etapas permaneceram com os mesmos funcionários. Nota-se com isso que o tempo gasto diminuiu consideravelmente, contribuindo assim para que as atividades do produto $Z$ sejam realizadas no tempo disponível. 
O gerenciamento da produção segundo a Teoria das Restrições: uma aplicação em uma empresa de desenvolvimento de software Ana Maria Magalhães Correia, Armistrong Martins da Silva, Ana Carolina Costa de Oliveira, Antônio de Mello Villar

\section{Passo 3 - Subordinar qualquer outro evento à decisão anterior:}

A terceira etapa consiste em subordinar todas as outras atividades às restrições. Isto significa que todas as demais atividades da empresa como, por exemplo, levantamento de processos, instalação de banco de dados e pré-configurações deverão trabalhar no ritmo das restrições. Nesse caso, deve-se deixar as demais atividades com capacidade ociosa. Assim, uma subordinação direta da decisão anterior é atender parcialmente a demanda do produto, ou seja, atender apenas 2 unidades por ano do produto Z, pautando-se unicamente no aspecto econômico-financeiro. Nessas condições não se analisa aspectos mercadológicos.

Em seguida, na empresa estudada optou-se em programar as restrições com a utilização de mais funcionários trabalhando e com isso agilizando os tempos das atividades conforme já explanado na tabela 4 e posteriormente fez-se a programação das demais atividades para que trabalhassem balanceadas com os fluxos das restrições encontradas. O passo seguinte é a elevação da restrição.

\section{Passo 4 - Elevar as restrições do sistema:}

A quarta etapa consiste em elevar a restrição do sistema. Elevar nesse caso, significa aumentar a capacidade para um nível mais alto (COX III; SPENCER, 2002). Assim na empresa estudada, decide-se portanto aumentar a capacidade das atividades de conversão de dados e personalizações com investimento na melhoria dos equipamentos, investimento no aumento da quantidade e na mudança de funcionários especializados, com horas extras e outro turno para aumentar a capacidade de produção e assim atender a demanda total de 3 unidades por ano do produto Z. Com isso, o tempo disponível dessas atividades vai aumentar, entretanto com as despesas operacionais aumentando de $\mathrm{R} \$ 510.915,29$ para $\mathrm{R} \$ 553.617,12$ e o custo do material direto de $\mathrm{R} \$ 42.646,96$ para $\mathrm{R} \$ 45.121,30$.

Desta forma, o novo fluxo de produção é alterado com essas modificações. Nesse caso, uma nova tabulação em função desses investimentos é demonstrada na tabela 5 . 
O gerenciamento da produção segundo a Teoria das Restrições: uma aplicação em uma empresa de desenvolvimento de software Ana Maria Magalhães Correia, Armistrong Martins da Silva, Ana Carolina Costa de Oliveira, Antônio de Mello Villar

Tabela 5 - Quantidade de horas x recursos após a utilização do 4 passo da TOC

\begin{tabular}{|c|c|c|c|c|c|c|}
\hline $\begin{array}{l}\text { Nome da } \\
\text { atividade }\end{array}$ & Produto & Demanda & $\begin{array}{c}\text { Tempo } \\
\text { (minutos) }\end{array}$ & $\begin{array}{c}\text { TempoTotal } \\
\text { (minutos) }\end{array}$ & $\begin{array}{c}\text { Disponível } \\
\text { (minutos) }\end{array}$ & $\begin{array}{c}\% \\
\text { Ocupação }\end{array}$ \\
\hline $\begin{array}{c}\text { Levantamento de } \\
\text { processos }\end{array}$ & \multirow{6}{*}{ Z } & \multirow{6}{*}{3} & 4.220 & 12.660 & 14.850 & 85,25 \\
\hline $\begin{array}{l}\text { Instalação banco } \\
\text { de dados e pré- } \\
\text { configurações }\end{array}$ & & & 342 & 1.026 & 2.160 & 47,50 \\
\hline $\begin{array}{c}\text { Conversão de } \\
\text { dados }\end{array}$ & & & 2. 550 & 7. 650 & 15.135 & 50,55 \\
\hline $\begin{array}{l}\text { Configurações e } \\
\text { personalizações }\end{array}$ & & & 7.796 & 23.388 & 25.845 & 90,49 \\
\hline Diversos & & & 1.985 & 5.955 & 7.245 & 82,19 \\
\hline Personalizaçōes & & & 18. 510 & 55.530 & 68.835 & 80,67 \\
\hline
\end{tabular}

Fonte: Dados da Pesquisa (2009).

As atividades de conversão de dados que antes apresentavam utilização de 12.240 minutos gastos, entretanto com disponibilidade de apenas 10.545 minutos e a atividade de personalizações com a utilização de 63.090 minutos gastos, entretanto com disponibilidade de apenas 61.275 minutos, passam a obter após o quarto passo de focalização da TOC, 7.650 minutos e 55.530 minutos, respectivamente.

Logo, verifica-se que com essas modificações propostas pela TOC, o quadro atual da empresa não apresenta restrições em nenhuma das atividades do processo produtivo do produto Z. Neste sentido, como a capacidade da restrição determina a capacidade do sistema, verifica-se agora o lucro máximo anual obtido após a utilização dos 4 passos da TOC, atendendo com isso a quantidade de 3 unidades do produto $Z$ demandas pelo mercado, com acréscimo das despesas operacionais e acréscimo do material direto como mostra a tabela 6. 
O gerenciamento da produção segundo a Teoria das Restrições: uma aplicação em uma empresa de desenvolvimento de software Ana Maria Magalhães Correia, Armistrong Martins da Silva, Ana Carolina Costa de Oliveira, Antônio de Mello Villar

Tabela 6 - Lucro máximo anual após o quarto passo da TOC

\begin{tabular}{|c|c|c|c|c|c|}
\hline Produto & Demanda & $\begin{array}{l}\text { Preço de } \\
\text { Venda } \\
\text { (R\$) }\end{array}$ & $\begin{array}{l}\text { Material } \\
\text { direto } \\
(\mathrm{R} \$)\end{array}$ & $\begin{array}{l}\text { Margem de Contribuição } \\
\text { (R\$) }\end{array}$ & $\begin{array}{c}\text { Lucro } \\
\text { (R\$) }\end{array}$ \\
\hline \multirow[t]{4}{*}{ Z } & 3 & $1.000 .000,00$ & $45.121,30$ & $954.878,70$ & $2.864 .636,10$ \\
\hline & & & & Ganho & $2.864 .636,10$ \\
\hline & & & & Despesas Operacionais & $553.617,12$ \\
\hline & & & & Lucro Máximo Anual & $2.311 .018,98$ \\
\hline
\end{tabular}

Fonte: Dados da Pesquisa (2009).

O lucro máximo real com a elevação das restrições é de $R \$ 2.311 .018,98$, portanto o ganho em relação ao lucro máximo anterior $(R \$ 1.657 .944,51)$ é de $R \$$ $653.074,47$. Ou seja, esse ganho se deu com a utilização da capacidade demandada pelo mercado ( 3 unidades por ano), juntamente com um aprimoramento do processo produtivo, identificação das restrições e uma melhor operacionalização das atividades, sendo possível otimizar a produção do sistema como um todo.

Passo 5 - Se no passo 4 as restrições são quebradas, volte ao passo 1 , mas não permita que a inércia tome conta do sistema:

A quinta e última etapa do processo de focalização das cinco etapas consiste em evitar que a inércia interrompa o processo de aprimoramento contínuo (COX III; SPENCER, 2002). Assim, na empresa estudada, apesar de ter resolvido o problema, cabe ainda verificar o quinto passo do processo de aprimoramento: se no passo anterior uma restrição foi quebrada, volte à primeira etapa, mas não deixe que a inércia cause nova restrição no sistema. Esse processo é cíclico e deve ser uma constante no gerenciamento da produção da empresa.

\section{CONSIDERAÇÕES FINAIS}

A Teoria das Restrições (TOC) tem como fator principal a identificação dos recursos que restringem a capacidade produtiva e a consequente aplicação dos cinco passos de focalização, sendo considerada como o ponto chave para a implementação de ações que otimizem o processo de produção e que levem a empresa ao alcance de 
O gerenciamento da produção segundo a Teoria das Restrições: uma aplicação em uma empresa de desenvolvimento de software Ana Maria Magalhães Correia, Armistrong Martins da Silva, Ana Carolina Costa de Oliveira, Antônio de Mello Villar

sua meta, que é ganhar dinheiro, através de três instrumentos: a margem de contribuição, os investimentos e as despesas operacionais.

Diante destes aspectos abordados pela TOC, este trabalho teve como objetivo investigar os benefícios da aplicação dos seus fundamentos no gerenciamento da produção numa empresa de desenvolvimento de software. Evidenciou-se nessa pesquisa que os principais pontos que são abordados pela teoria servem como norteadores no processo decisório desta entidade, tais como a utilização dos cinco passos de focalização da TOC no processo de aprimoramento contínuo da empresa.

Seguindo os cinco passos da Teoria das Restrições, foram identificadas nas atividades da empresa as duas restrições do sistema (conversão de dados e personalizações); a quantidade de tempo das restrições foi utilizada em sua totalidade; as demais atividades foram subordinadas às restrições; e a elevação da restrição foi proporcionada com investimentos na melhoria dos equipamentos, na quantidade de funcionários especializados e na mudança desses funcionários nas etapas do processamento das atividades que apresentaram as restrições, obtendo com isso, ganhos de tempo essenciais para a solução dos problemas da empresa e 0 atendimento total da demanda de 3 unidades do produto $Z$ por ano. Deve-se salientar também, o ganho obtido, pois, o lucro líquido passou de $R \$ 1.657 .944,51$ para $R \$$ 2.311.018,98, com um aumento de $71,74 \%$.

Desta forma, os benefícios esperados dessa aplicação citados no início desta pesquisa, foram alcançados, quando a partir dos cinco passos de focalização da TOC, obteve-se: i) melhor compreensão de todo o processo produtivo da empresa; ii) uma visão sistêmica dos problemas encontrados; e por consequência; iii) um melhor gerenciamento desse processo, buscando a priorização das soluções que melhor se adequassem as necessidades da empresa.

Portanto, os resultados encontrados permitem concluir que a Teoria das Restrições é uma ferramenta de melhoria contínua bastante útil no gerenciamento da produção e que o uso de suas medidas de desempenho verifica-se bem viável e promissor. Os gestores têm que estar cientes de que sempre existe, no mínimo, uma restrição e, a partir de aí identificá-la e trabalhá-la como uma oportunidade de alavancar 
O gerenciamento da produção segundo a Teoria das Restrições: uma aplicação em uma empresa de desenvolvimento de software Ana Maria Magalhães Correia, Armistrong Martins da Silva, Ana Carolina Costa de Oliveira, Antônio de Mello Villar

o ganho total da empresa. Para isso, fez-se necessária uma integração destas medidas, com o envolvimento de todas as áreas de produção da empresa na verificação da identificação de ações a serem realizadas para otimização do sistema como um todo.

\section{REFERÊNCIAS}

BORNIA, Antônio Cézar. (2009). Análise gerencial de custos: aplicação em empresas modernas. (2 ed.). São Paulo: Atlas.

CABRAL, A. C. D.; FLEURY, A. C. C. (2007). Competitividade Sistêmica: um modelo de análise de cenários para gestão de empresas. Revista Gestão Industrial. v. 03. Ponta Grossa, Paraná.

CORBETT NETO, T. (1997). Contabilidade de Ganho: a nova abordagem gerencial de acordo com a teoria das restrições. São Paulo: Nobel.

COX III, J. F.; SPENCER, M. S. (2002). Manual da Teoria das Restrições. Trad. Fernanda Kohmann Dietrich. Porto Alegre: Boockman.

DETTMER, H. W. (1997). Goldratt's Theory of Constraints. ASQC Quality Press.

ELMAGHRABY, S. E. E. et al. (2003). Note on the paper 'Resource-constrained project management using enhanced theory of constraint by, Wei et al. International Journal of Project Management. 21 301-305.

FAVARETTO, F. (2001). Uma contribuição ao processo de gestão da produção pelo uso da coleta automática de dados de chão de fábrica. São Carlos, SP. (Tese de Doutorado). Escola de Engenharia de São Carlos, Universidade de São Paulo - USP, 253p.

GOLDRATT, E. M. (1992). A corrida pela vantagem competitiva. (2 ed.). São Paulo: IMAN. ed.). São Paulo: Educator.

; COX, J. (1997). A meta: um processo de aprimoramento contínuo. (12

GUPTA, M.; et al. (2002). TOC- based performance measures and focusing steps in a job-shop manufacturing environment. International Journal of Production Research, v. 40, n. 4, p. 907-930. 
O gerenciamento da produção segundo a Teoria das Restrições: uma aplicação em uma empresa de desenvolvimento de software Ana Maria Magalhães Correia, Armistrong Martins da Silva, Ana Carolina Costa de Oliveira, Antônio de Mello Villar

KIM, K. H.; et al. (1997). A negotiation based scheduling for items with flexible process plan. Computers in Industrial Engineering, n. 3-4, vol.33, p. 785-788.

KOPAK, S. C. (2006). Modelo conceitual de sistema de gestão da produção baseado na Teoria das Restrições. In: XXVI Encontro Nacional de Engenharia da Produção, Fortaleza - CE. Anais...Fortaleza, ENEGEP.

MARTINS, E. (2001). Contabilidade de custos. (8 ed.). São Paulo: Atlas.

NARDINI, J. J.; PIRES, S. R. I. (2002). Tecnologias e sistemas de gestão da produção e da qualidade: um estudo na indústria metal-mecânica. In: XXII Encontro Nacional de Engenharia de Produção, Curitiba - PR. Anais...Curitiba, ENEGEP.

NOREEN, E.; et al. (1996). A teoria das restrições e suas implicações na contabilidade gerencial. São Paulo: Educador.

ROGERS, P.; REIS, E. A. (2005). Teoria das Restrições e Decisões de Longo Prazo: Caminho para a Convergência. In: V Congresso USP de Controladoria e Contabilidade, São Paulo - SP. Anais...São Paulo, USP.

SILVA, J. A. B.; et al. (2005). As medidas de desempenho como uma ferramenta de gerenciamento. In: XII Congresso Nacional de Estudantes de Engenharia Mecânica, Ilha Solteira - SP. Anais... Ilha Solteira.

SLACK, N.; et al. (2001). Administração da Produção. Edição compacta, São Paulo: Atlas.

TUBINO, D. F. (1997). Manual de Planejamento e Controle da Produção. São Paulo: Atlas.

Alegre: Boockman. (1999). Sistemas de produção: a produtividade no chão-de-fábrica. Porto

VILLAR, A. M.; et al. (2008). Planejamento, Programação e Controle da Produção. Editora Universitária da UFPB. João Pessoa.

WANKE, P. F. (2004). Teoria das Restrições: Principais Conceitos e Aplicação Prática. Revista Tecnologística, v.1, n. 99. São Paulo.

WEI, C. C.; et al. (2002). Resource-constrained project management using enhanced theory of constraint. International Journal of Project Management 20:561-7.

Data de Submissão: 02/12/2009

Data de Aceite: 29/10/2010 